\title{
Performance Comparison of Wavelets Generated from Four Different Orthogonal Transforms for Watermarking With Various Attacks
}

\author{
H. B. Kekre ${ }^{1}$, Tanuja Sarode ${ }^{2}$, Shachi Natu ${ }^{3}$ \\ ${ }^{1}$ MPSTME, Department of Computer Engineering, NMIMS University, Mumbai, India \\ hbkekre@yahoo.com \\ ${ }^{2}$ Department of Computer Engineering, TSEC, Mumbai University, India \\ tanuja_0123@yahoo.com \\ ${ }^{3} \mathrm{Ph}$. D. Research Scholar, MPSTME, NMIMS University, Mumbai, India \\ shachi_natu@yahoo.com
}

\begin{abstract}
This paper proposes a watermarking technique using different orthogonal wavelet transforms like Hartley wavelet, Kekre wavelet, Slant wavelet and Real Fourier wavelet transform generated from corresponding orthogonal transform. These orthogonal wavelet transforms have been generated using different sizes of component orthogonal transform matrices. For example $256^{\star} 256$ size orthogonal wavelet transform can be generated using $128^{\star} 128$ and $2^{\star} 2$ size component orthogonal transform. It can also be generated using $64^{\star} 64$ and $4^{\star} 4,32^{\star} 32$ and $8^{\star} 8,16^{\star} 16$ and $16^{\star} 16$ size component orthogonal transform matrices. In this paper the focus is to compare the performance of above mentioned transforms generated using $128^{*} 128$ and $2 * 2$ size component orthogonal transform and $64^{*} 64$ and $4 * 4$ size component orthogonal transform in digital image watermarking. The other two combinations are not considered as their performance is comparatively not as good. Comparison shows that wavelet transforms generated using $(128,2)$ combination of orthogonal transform give better performances than wavelet transforms generated using $(64,4)$ combination of orthogonal transformfor contrast stretching, cropping, Gaussian noise, histogram equalization and resizing attacks. Real Fourier wavelet and Slant wavelet prove to be better for histogram equalization and resizing attack respectively than DCT wavelet and Walsh wavelet based watermarking presented in previous work.
\end{abstract}

\section{KEYWORDS}

Watermarking, Slant wavelet, Kekre wavelet, Hartley wavelet, Real Fourier wavelet

\section{Council for Innovative Research}

Peer Review Research Publishing System

Journal: INTERNATIONAL JOURNAL OF COMPUTERS \& TECHNOLOGY

Vol 9, No 3

editor@cirworld.com

www.cirworld.com, member.cirworld.com 


\section{INTRODUCTION}

With the advent of digital era, use of internet to disseminate digital images and other multimedia contents is inevitable. This imposes an immense need of security of digital contents transmitted over network. Availability of various tools and techniques allows easy manipulation of digital contents. To protect the digital contents from such undesirable alterations was the motivation for watermarking techniques. Though cryptographic techniques are there to provide security to digital contents, they don't contribute in protecting copyright of content owner. Watermarking techniques are explicitly meant for protecting the identity of owner of digital contents so that no one else can claim the ownership and can alter the contents. Thus identity of owner in some or the other form is hidden into digital contents for digital authentication and copyright protection. Digital contents can be digital images, audio or video signals or text documents. Based on the contents to be protected, watermarking can be classified as digital image watermarking, audio watermarking, video watermarking or text watermarking. In case of digital image watermarking, some secret signal in the form of binary code or image known as watermark is inserted into digital image (host image) to be protected. This insertion process is usually termed as watermark embedding and after embedding the watermark into host image we get watermarked image. At the receiving end watermark can be removed from watermarked image only by authentic/ intended users and is called as watermark extraction. Any damage to the extracted watermark indicates illegal alterations to host image. Digital image watermarking is further classified into different streams based on different criteria. When watermark is embedded by altering pixel values of a host image, it is called as spatial domain watermarking. In contrast when watermark is embedded in the transformed image obtained by applying suitable transformation technique, it is called as transform domain watermarking. Another classification is based on whether the watermark embedded is visible to the observer or not. If watermark can be seen in watermarked image it is visible watermarking otherwise it is invisible watermarking. One more classification of digital image watermarking is based on necessary data for watermark extraction. If host image is required to perform watermark extraction, it is called as informed or private watermarking. If original image is not required for watermark extraction, it is called as blind or public watermarking.

Three major requirements for watermarking technique are:

- Imperceptibility which means presence of watermark in host image should not be noticeable. In other words, embedding watermark should not lead to significant loss of host image quality.

- Robustness means embedded watermark should not get destroyed due to intentional or non-intentional alterations made to host image.

- Security refers to the difficulty to remove watermark from host image for non-owners.

Transform domain watermarking techniques are more popular than spatial domain techniques due to their robustness but at the same time they are expensive in terms of complexity. Hence it has opened a new gateway for researchers to find more and more efficient transform domain watermarking techniques. In recent years, use of wavelet transforms for various image processing applications is at its peak and watermarking is not an exception. It has also motivated us to use different wavelet transforms for watermarking and to compare their performances.

Organization of paper is as follows. Section 2 presents related work in watermarking field. Section 3 focuses on Real Fourier transform, Slant transform Hartley transform and Kekre's transform used for generation of orthogonal wavelet transforms. Proposed watermarking technique is given in Section 4. Section 5 contains discussion on the results of proposed technique. Finally the paper is concluded in Section 6.

\section{RELATED WORK}

As stated earlier, transform domain watermarking techniques are more robust and hence gained more popularity. In literature we find many such transform based watermarking techniques. Discrete Cosine Transform (DCT) $\left[1,{ }^{2},{ }^{3}\right]$, Discrete Fourier Transform (DFT)[4,5], Discrete Wavelet Transform (DWT)[6,7,8], Singular Value Decomposition (SVD)[8,9] are some of popular transformation techniques used in watermarking. These transformation techniques can be used independently or in combination with each other for watermarking. Use of combined or multiple transforms together has proven to be more robust than use of any single transform for watermarking. Khaled Loukhaoukhaand Jean-Yves Chouinard[10] have proposed a non-blind robust watermarking algorithm for grayscale image. Binary code is used as watermark. Wavelet coefficients of host image are computed up to three levels and then watermark is embedded in $\mathrm{HH}$, $\mathrm{LH}$ and $\mathrm{HL}$ sub-bands using thresholding coefficients. $\mathrm{HH}, \mathrm{LH}$ and $\mathrm{HL}$ sub-bands are selected for embedding process because Human Visual System (HVS) is less sensitive to small changes in texture and edges of an image. Threshold values are computed for each sub-band except $L L$ band and watermark is embedded if the matrix entry for each sub-band is greater or equal to threshold value. Yong-Gang Fu and Hui-RongWang [11] have proposed another wavelet transform based digital watermarking scheme wherein a random binary image is obtained by random permutations. This watermark is then embedded in two level decomposed host images using bi-orthogonal spline wavelet transform. Authors have proposed two different schemes to compute watermark strength controlling factor. In first scheme, this factor is kept constant whereas in second scheme, it is computed in adaptive manner depending on the detail coefficients at the horizontal and vertical direction of the level second waveletdecomposition. First scheme is easy to use whereas second scheme causes less degradation of host image. In [12], authors proposed a watermarking scheme for colour images by embedding colour image into it. Watermark is converted into binary stream by DCT transforming it and then quantifying it. Host image is converted from RGB colour space to $Y I Q$ colour space. Luminance $Y$ is then subjected to Integer wavelet Transform Waveletcoefficients of host image arethen quantified to watermark information. This makes the algorithm more robust and also original watermark and host image arenot required for watermark extraction. In [13], authors have proposed SVD and wavelet transform based watermarking method. Here a binary watermark is embedded in singular 
values of selected wavelet coefficients of first level vertical and horizontal sub-bands of grayscale image. Combination of SVD and DWT makes it robust against common image processing attacks like JPEG compression, cropping, low pass filtering and Gaussian noise attack.Gurparkash Singh Kang in [14] have proposed an adaptive blind watermarking technique in which low frequency components of DCT transformed watermark are embedded in wavelet coefficients of LL band of host image. Qualified significant wavelet tree are used to select the locations for embedding and extracting watermark.

\section{REAL FOURIER TRANSFORM[15]:}

Discrete Fourier Transform (DFT) contains complex exponentials and gives complex values in output. To avoid these complex values in output of DFT, Cosine and Sine coefficients can be used. DCT and DST both are orthogonal even after sampling and are equivalent to FFT of data sequence of double length. Discrete Cosine Transform uses real and even functions while Discrete Sine Transform uses real and odd functions. Hence by using coefficients of DCT and DST, we can obtain Fourier transform containing only real coefficients. Since it contains only real terms, it is called as Real Fourier transform or Sine Cosine transform. Once this Real Fourier transform is obtained from DCT and DST matrices, its wavelet can be generated using the procedure mentioned in [16].

\section{SLANT TRANSFORMAND HARTLEY TRANSFORM[17]:}

Slant transform matrix is orthogonal with first basis function constant and linear second basis function. The unitary kernel matrix of $2 * 2$ Slant matrix is obtained from $2 * 2$ Haar or Hadamard transform.

$$
\mathrm{H}_{\mathrm{N}}(2)=1 / \sqrt{ } 2\left[\begin{array}{cc}
1 & 1 \\
1 & -1
\end{array}\right]
$$

Subsequent Slant transform matrices are generated in iterative manner such that the step size is uniform and resultant transform matrix is orthogonal. This iterative procedure is actually a product of two sparse matrices and hence results in faster transform.

Hartley transform jointly uses the sine and cosine basis functions but its coefficients are real and not complex like Fourier transform which also uses sine and cosine functions. The two dimensional Discrete Hartley transform is given by

$$
F(u, v)=\frac{1}{N} \sum_{x=0}^{N-1} \sum_{y=0}^{N-1} f(x, y) \operatorname{cas}\left\{\frac{2 \pi}{N}(u x+v y)\right\}
$$

The inverse Hartley transform is given by

$$
f(x, y)=\frac{1}{N} \sum_{u=0}^{N-1} \sum_{v=0}^{N-1} F(u, v) \operatorname{cas}\left\{\frac{2 \pi}{N}(u x+v y)\right\}
$$

Where $\operatorname{cas} \theta=\cos \theta+\sin \theta$

\section{KEKRE TRANSFORM}

Kekre transform matrix of size $\mathrm{N}^{*} \mathrm{~N}$ is given as follows:

$$
K_{x y}=\left\{\begin{aligned}
1, & x<y \\
-N+(x-1), & x=y+1 \\
0, & x>y+1
\end{aligned}\right.
$$

An added advantage of Kekre transform over other orthogonal transforms is its size. Unlike other transforms, Kekre transform need not be of size which is power of 2 . Hence it is easily applicable to image of any size. Kekre wavelet transform is generated from Kekre transform using procedure in [18]. This procedure is slightly different than the generation of other orthogonal wavelet transforms. Kekre wavelet transform also can be of any size which is multiple of $\mathrm{N}$ where $\mathrm{N}$ is the size of Kekre transform matrix.

\section{PROPOSED METHOD}

In this section, watermarking technique using orthogonal wavelet transforms followed by DCT and SVD is proposed. These techniques have been implemented on 1.33 GHz AMD Dual Core Processor with 4 GB RAM and MATLAB 7.2.

In the proposed method, $256^{*} 256$ size colour host images and $128^{*} 128$ size watermarks are used. A test bed of 10 colour host images and 5 colour watermarks has been used. Wavelet transforms of size $256^{\star} 256$ and $128^{\star} 128$ are generated using two pairs of component orthogonal transforms, 1) $128^{*} 128$ and $2 * 2$ size orthogonal transform matrices and 2) $64 * 64$ and $4 * 4$ size orthogonal transform matrices. Hartley wavelet, Slant wavelet and Real Fourier wavelet transforms are generated using procedure in [16] and Kekre wavelet transform is generated using procedure in [18]. 


\section{Watermark Embedding Algorithm:}

Step 1.Take two level wavelet transform of Red, Green and Blue channel of host image using $256^{\star} 256$ wavelet transform matrix.

Step 2. Apply DCT to HL2 sub-band and arrange the resultant coefficients in zigzag manner to get four quadrants out of it. These four quadrants are used to embed watermark multiple times in host image.

Step 3. Obtain singular values of each quadrant by applying SVD to them.

Step 4.Perform similar steps on watermark to get their singular values. Use $128 * 128$ wavelet transform matrix for watermarks.

Step 5. Alter the singular values of host image by adding scaled singular values of watermark to them.

$$
\mathrm{S}^{\prime \prime}=\mathrm{S}+\mathrm{KS} \mathrm{S}^{\prime}
$$

Where, $S$ is the singular value matrix of each quadrant, $S^{\prime}$ is the singular value matrix of watermark and $S^{\prime \prime}$ is the modified singular value matrix of host image.

Step 6. Reconstruct the watermarked image by following inverse zigzag, inverse DCT and inverse 2-level wavelet transform in sequence.

Step 7.Calculate Mean Absolute Error (MAE) between host image and watermarked image as a measure of imperceptibility.

\section{Watermark Extraction Algorithm}

Step 1.Take two level wavelet transform of Red, Green and Blue channel of watermarked image using $256^{\star} 256$ wavelet transform matrix.

Step 2.Apply DCT to HL2 sub-band and arrange the resultant coefficients in zigzag manner to get four quadrants out of it. From these four quadrants watermark is extracted from watermarked image.

Step 3.Obtain singular values of each quadrant by applying SVD to them.

Step 4. Extract singular values of watermark from singular values of watermarked image and singular values of host image.

$$
\mathrm{S}^{\prime}=\left(\mathrm{S}^{\prime \prime}-\mathrm{S}\right) / \mathrm{K}
$$

Where, $S$ is the singular value matrix of each quadrant, $S^{\prime}$ is the singular value matrix of watermark and $S^{\prime \prime}$ is the modified singular value matrix of host image.

Step 5. Construct DCT coefficients of watermark using the singular values extracted in Step 4.

Step 6. Extract the watermarks from watermarked image by following inverse DCT and inverse 2-level wavelet transform in sequence.

Step 7. Calculate Mean Absolute Error (MAE) between original watermark and extracted watermark as a measure of robustness.

Figure 1 and Figure 2 show the test set of ten host images and five watermarks used for experimental work.

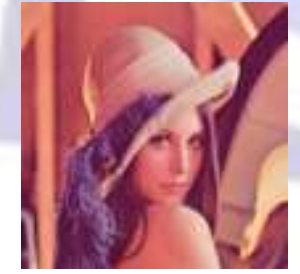

(a)

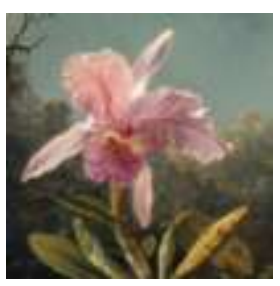

(f)

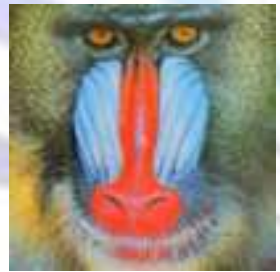

(b)

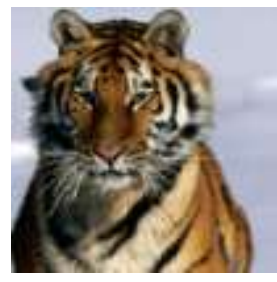

(g)

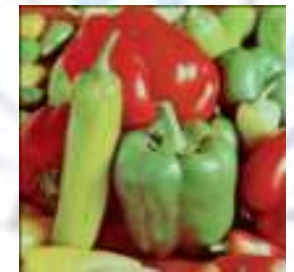

(c)

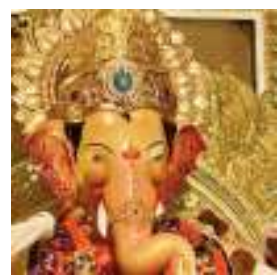

(h)

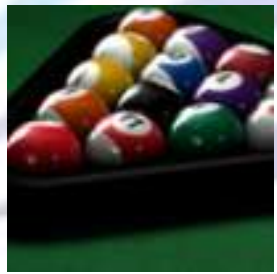

(d)

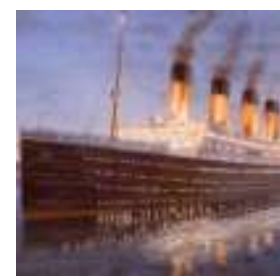

(i)

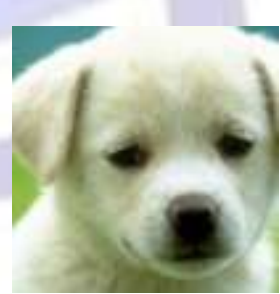

(e)

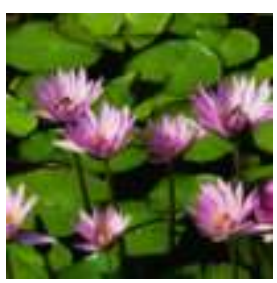

(j)

Figure 1. Cover images used for experimentation (a)Lena (b)Mandrill (c)Peppers (d)Balls (e)Puppy (f)Tiger (g)Flower (h)Ganesh (i)Titanic (j)Waterlili 


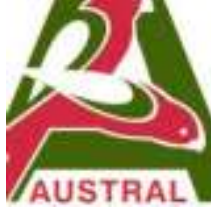

(a)

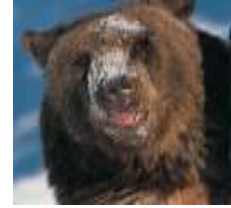

(b)

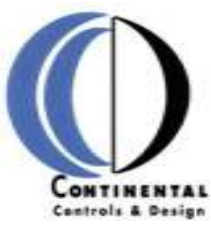

(c)

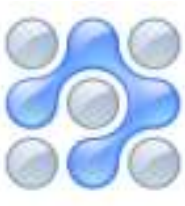

(d)

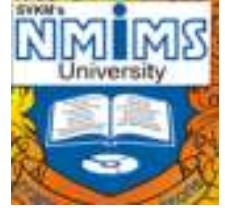

(e)

Figure 2. Watermark images used for experimentation (a) Austral (b) Bear (c) CCD (d) Logo (e) NMIMS

\section{RESULTS AND DISCUSSION:}

Sample result images are shown in Table 1 and Table 2 for Slant wavelet and Real Fourier wavelet transform respectively.

Table 1 below shows the watermarking result images for Slant wavelet obtained from component orthogonal Slant transform matrices of size $128^{\star} 128$ and $2^{\star} 2$. These result images are for host image 'peppers' and 'ccd' watermark with scaling factor 0.6. Watermarked image without performing any attack is shown with Mean Absolute Error value between host and watermarked image in first row. In the following rows, watermarked images with different image processing attacks on them are shown with corresponding Mean Absolute Error value between watermarked images and attacked watermarked image. For both the cases, watermarks extracted from four quadrants of HL2 sub-band are shown. Mean Absolute Error between original watermark and extracted watermark from each quadrant are listed below them.

Table 1. Result images for Slant wavelet obtained from component orthogonal Slant transform matrices of size $128^{\star} 128$ and $2 * 2$. (Host image $=$ 'pepper', watermark= 'ccd', $\left.k=0.6\right)$

\begin{tabular}{|c|c|c|c|c|c|c|}
\hline \multirow{2}{*}{ Type of Attack } & \multirow{2}{*}{$\begin{array}{c}\text { Host Image/ } \\
\text { Watermarked } \\
\text { Image }\end{array}$} & \multirow{2}{*}{$\begin{array}{l}\text { Watermarked } \\
\text { Image / Image } \\
\text { after } \\
\text { performing } \\
\text { attack }\end{array}$} & \multicolumn{4}{|c|}{ Watermarks Extracted from four quadrants } \\
\hline & & & Q1 & Q2 & Q3 & Q4 \\
\hline \multicolumn{7}{|l|}{ No attack } \\
\hline Mean Absolute Error & & 10.647 & 9.490 & 9.501 & 9.506 & 9.511 \\
\hline \multicolumn{7}{|l|}{ Contrast Stretching } \\
\hline Mean Absolute Error & & 25.82751 & 10.650 & 10.769 & 10.745 & 10.829 \\
\hline \multicolumn{7}{|l|}{ Cropping } \\
\hline Mean Absolute Error & & 15.981 & 10.002 & 10.361 & 10.278 & 10.230 \\
\hline \multicolumn{7}{|l|}{ Gaussian Noise } \\
\hline Mean Absolute Error & & 29.193 & 9.937 & 9.800 & 9.826 & 9.960 \\
\hline
\end{tabular}




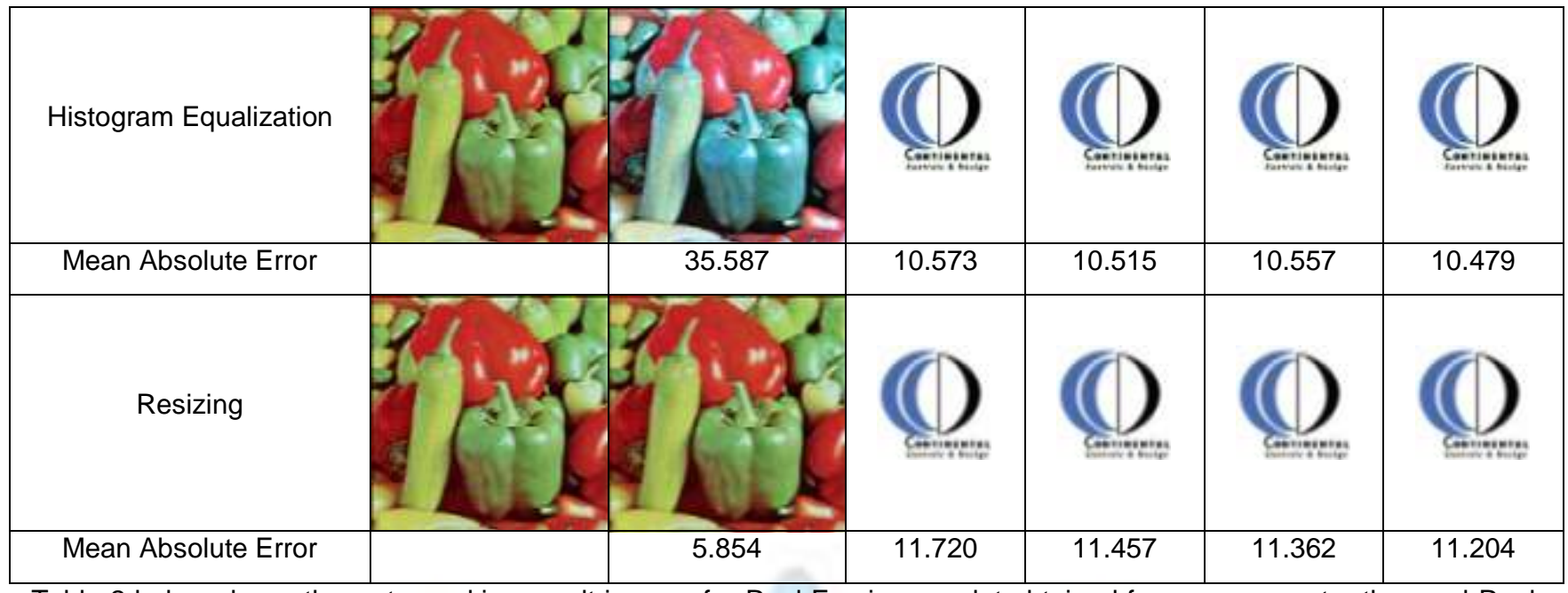

Table 2 below shows the watermarking result images for Real Fourier wavelet obtained from component orthogonal Real Fourier transform matrices of size $64^{*} 64$ and $4^{*} 4$. These result images are for host image 'balls' and 'logo' watermark with scaling factor 0.6. Watermarked image without performing any attack is shown with Mean Absolute Error value between host and watermarked image in first row. In the following rows, watermarked images with different image processing attacks on them are shown with corresponding Mean Absolute Error value between watermarked images and attacked watermarked image. For both the cases, watermarks extracted from four quadrants of HL2 sub-band are shown. Mean Absolute Error between original watermark and extracted watermark from each quadrant are listed below them.

Table 2. Result images for Real Fourier wavelet obtained from component orthogonal Real Fourier transform matrices of size $64^{\star} 64$ and $4^{*} 4$ (host image= 'balls', watermark= 'logo', $k=0.6$ )

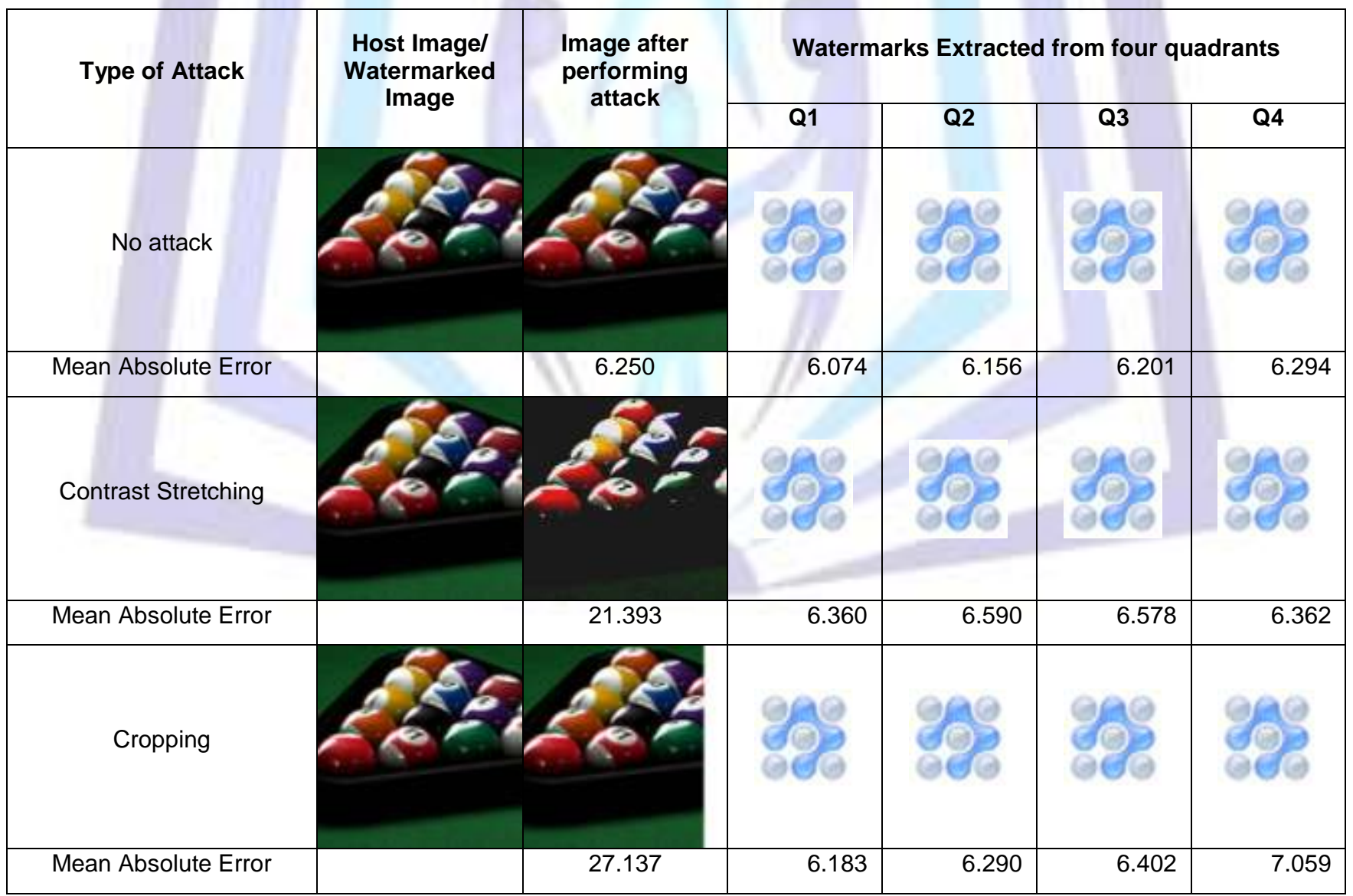




\begin{tabular}{|l|l|l|l|l|l|}
\hline Gaussian Noise & & & & & \\
\hline
\end{tabular}

Since ten host images and five watermarks are used to perform experimental work, comparison of various wavelet transforms used in watermarking has been done by taking average Mean Absolute Error of ten host images for each watermark. Further, average Mean Absolute Error of five such watermarks is calculated to represent MAE given by individual wavelet transform. Figure 3 below shows the comparison of all wavelet transforms when watermark is simply embedded in host image and watermarked image is not subjected to any kind of attack.

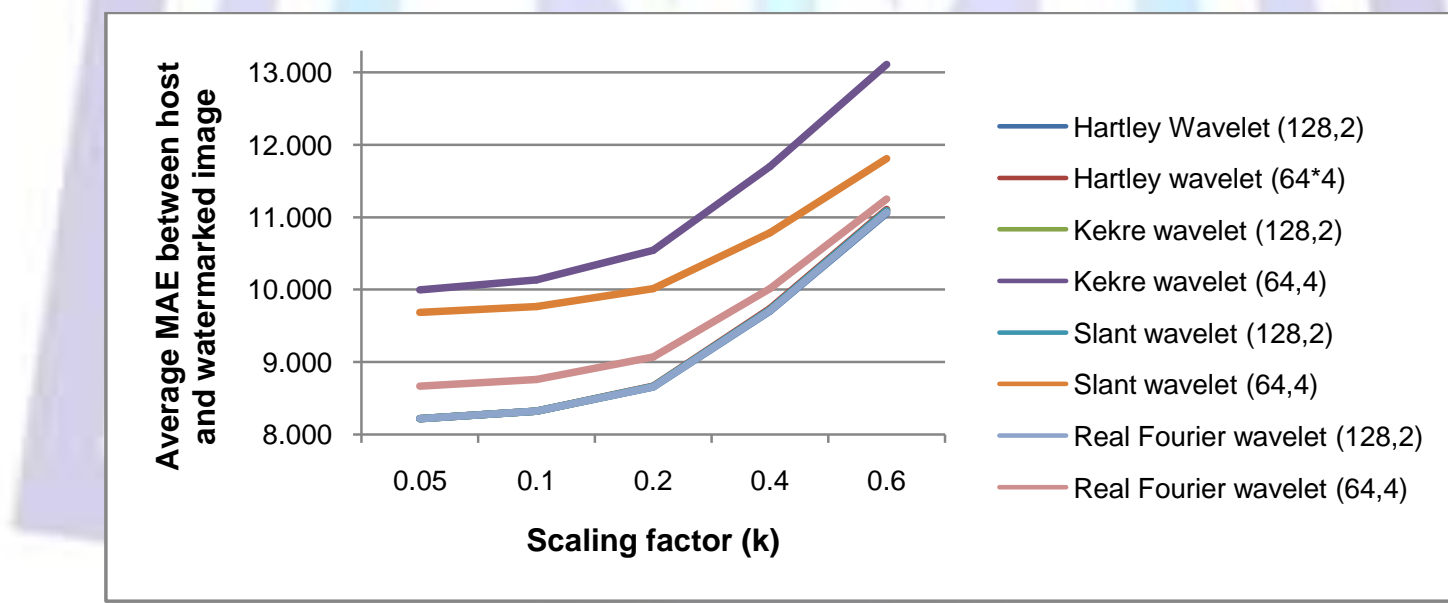

\section{Figure 3. Performance comparison of different wavelet transforms in watermark embedding with MAE against} scaling factor values

From above Figure 3, it can be clearly seen that as scaling factor $(k)$ value increases from 0.05 to 0.6 , visual quality of watermarked image degrades. This reduces imperceptibility which is one of the major requirements of watermarking. The wavelet combination of $128^{\star} 128$ and $2^{\star} 2$ gives the best result for all transforms for values of $k$ varying from 0.05 to 0.6 closely followed by Hartley wavelet transform of combination $(64,4)$.

Effect of increased scaling factor on various attacks performed on watermarked image for different wavelet transforms is shown in following figures. Figure 4 shows scaling factor against MAE value between original watermark and extracted watermark for different wavelet transforms. 


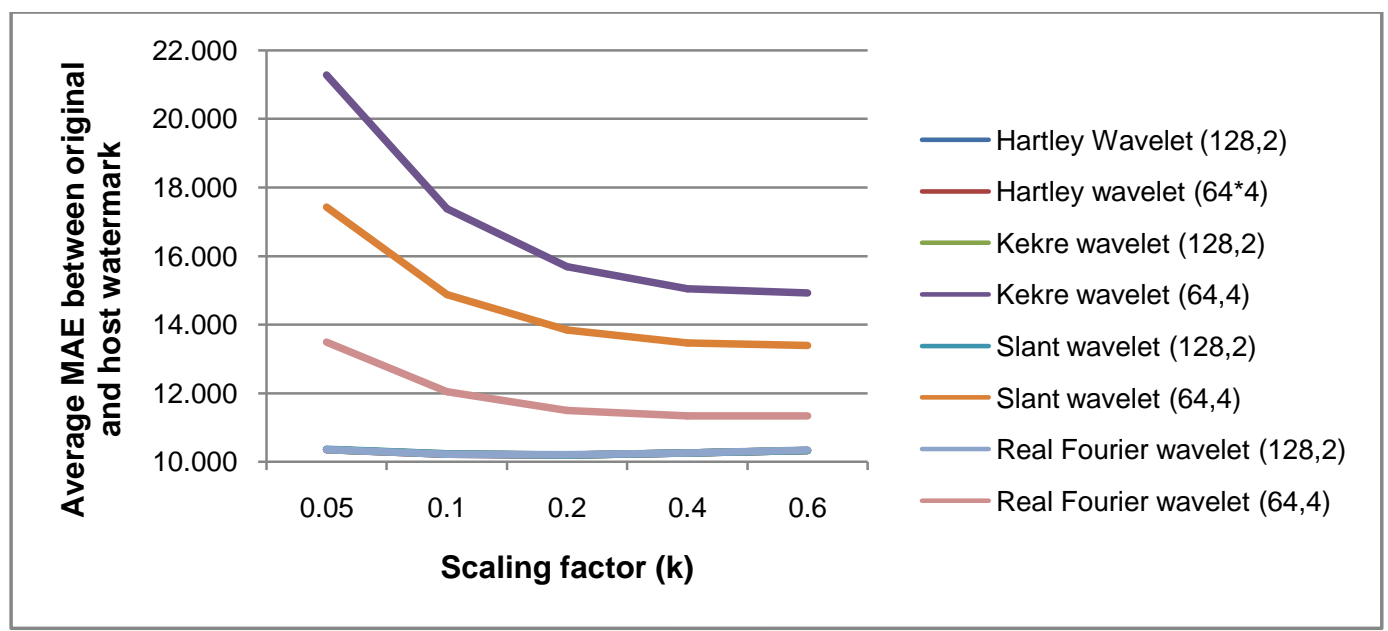

Figure 4. Average MAE between original and extracted watermark against scalingfactor for different wavelet transforms when no attack is performed on watermarked image.

From Figure 4 it can be observed that for higher scaling factor value, MAE between original and extracted watermark is reduced. All Wavelet transforms obtained from $128^{*} 128$ and $2 * 2$ component orthogonal transforms give best result for watermark extraction. Also Hartley transform with the combination of $(64,4)$ is the close follower.

Figure 5 shows the comparison of various wavelet transforms for different scaling factor values when contrast stretching attack is performed on watermarked image. Mean Absolute Error between watermarked image and attacked watermarked image is compared in Figure 5. Whereas Figure 6 shows Mean Absolute Error between original and extracted watermark against different scaling factor values for various wavelet transforms.

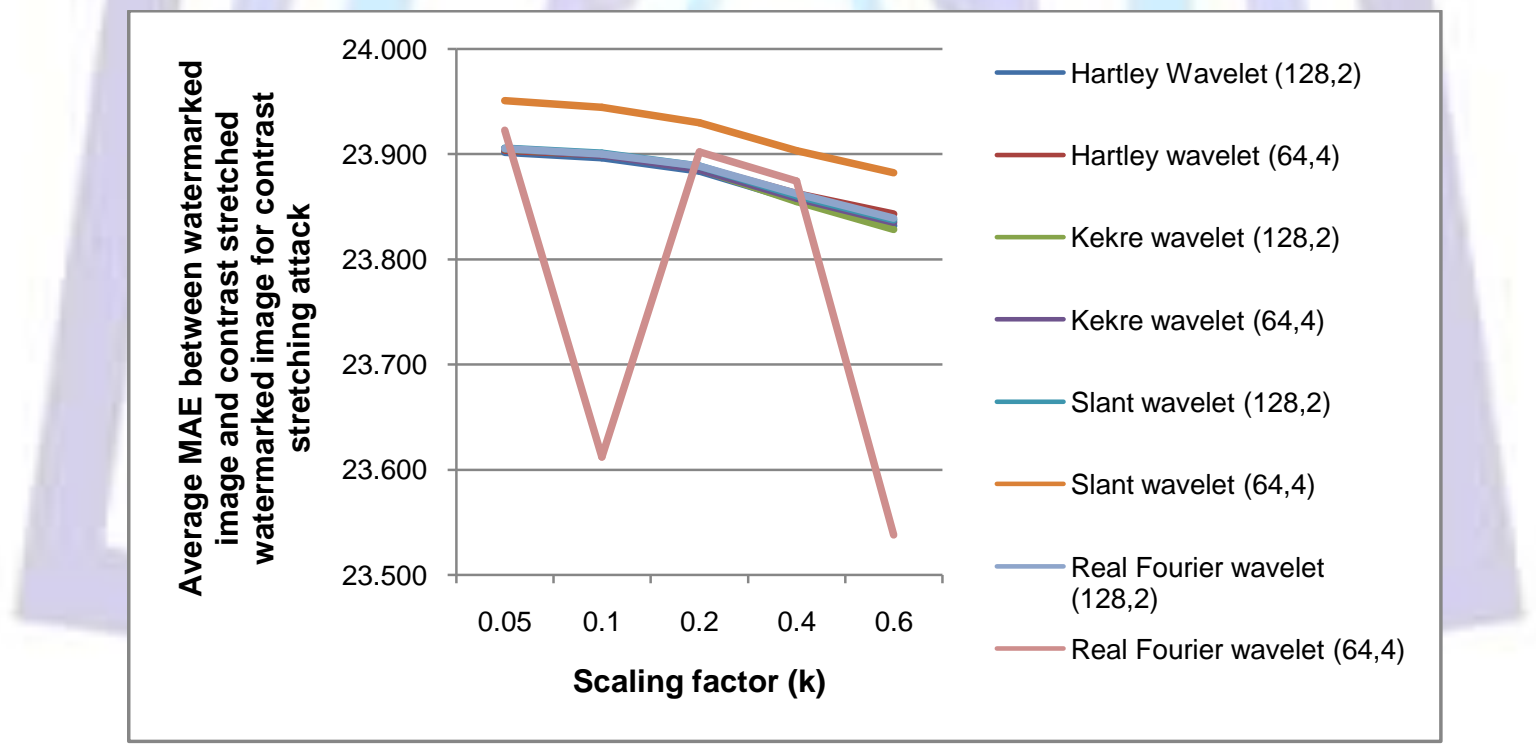

Figure 5. Performance comparison of different wavelet transforms in terms of Average MAE between watermarked image and contrast stretched watermarked image against different scaling factor values

From Figure 5 we can say that Average MAE between watermarked image and attacked watermarked image gradually reduces for contrast stretching attack except for Real Fourier transform where it shows continuous fluctuation. For all values of scaling factor, performance of Hartley wavelet, Kekre wavelet, Slant wavelet transform obtained from both combinations and Real Fourier wavelet obtained from $(128,2)$ combination are very close. Among them Kekre wavelet transform obtained from $(128,2)$ gives the best results. Although Real Fourier wavelet shows wide fluctuation, it gives the lowest error for all values of $k$ except a range of 0.2 to 0.4 . 


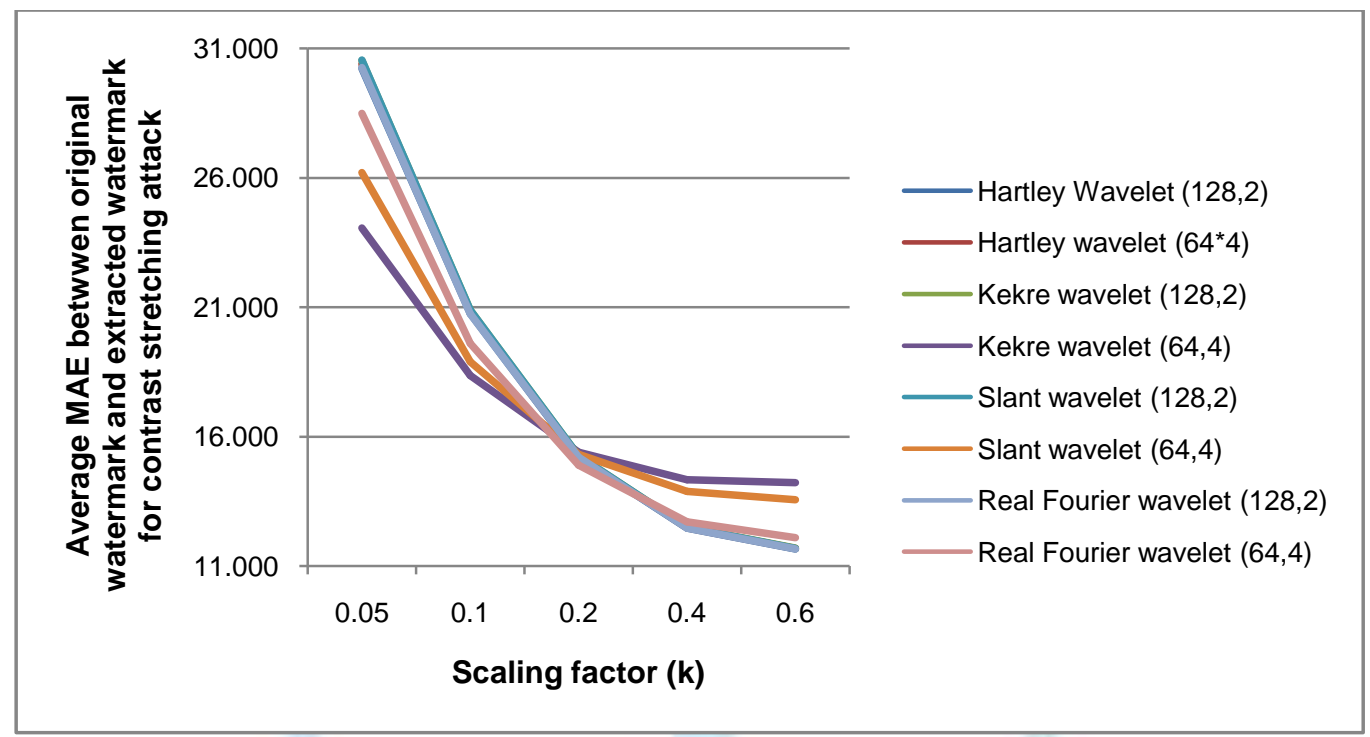

Figure 6. Average MAE between original and extracted watermark for different scaling factors and different wavelet transforms in contrast stretching attacks.

Figure 6 clearly indicates that for higher value of $\mathrm{k}$, better correlation between original and extracted watermark is observed. For the values of $\mathrm{k}$ ranging from 0.2 to 0.6 , all wavelet transforms with the combination of $(128,2)$ along with Hartley wavelet obtained from $(64,4)$ gives minimum error.Highervalue of scaling factor increases the robustness of proposed watermarking algorithm against contrast stretching attack.

Figure 7 shows the response of various wavelet transforms mentioned earlier against cropping attack. It shows average MAE between watermarked image and cropped watermarked image. Unlike contrast stretching attack,the average MAE between watermarked images before and after the attack is found to be increasing with increasing value of scaling factor.Kekre wavelet transform obtained from $64^{*} 64$ and $4^{*} 4$ Kekre transforms gives minimum distortion in cropped watermarked image.

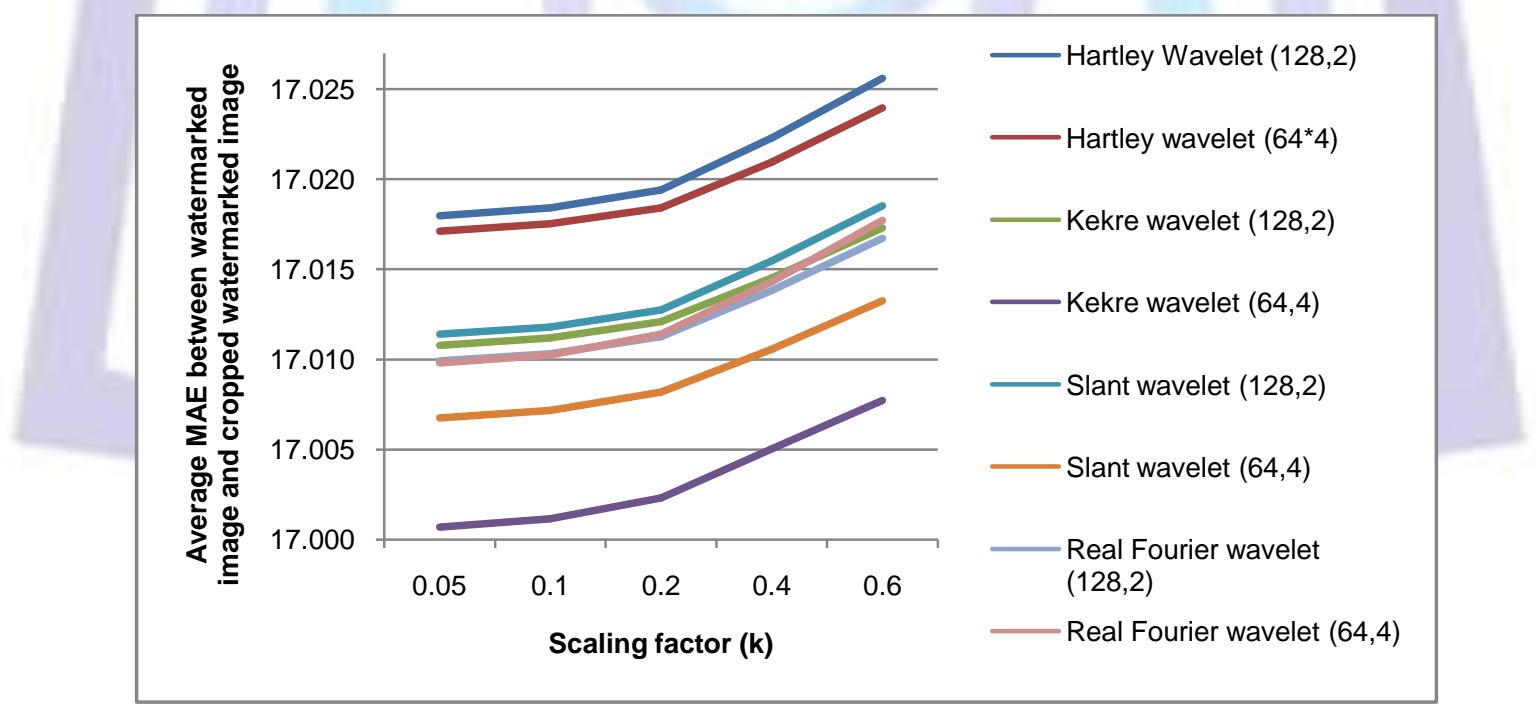

Figure 7. Average MAE between watermarked image and cropped watermarked image for different scaling factors and wavelet transforms 
For cropping attack, average MAE between original and extracted watermarks against scaling factor values are plotted in Figure 8.

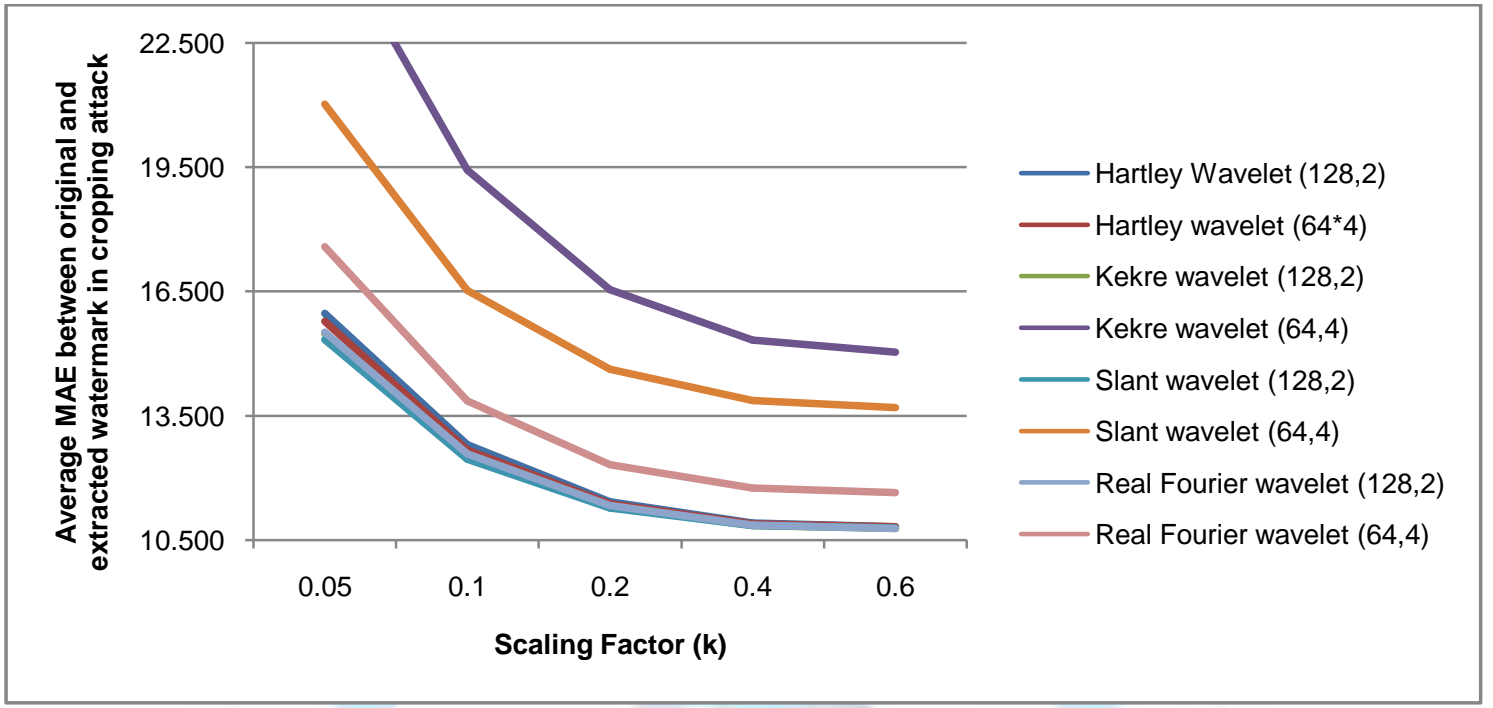

Figure 8. Average MAE between original watermark and extracted watermark for image cropping attack against scaling factor

Once again all wavelet transforms obtained from $(128,2)$ combination along with Hartley wavelet transform obtained from $(64,4)$ combination give minimum error for all values of $\mathrm{k}$. amongst them Real Fourier wavelet transform generated from $(128,2)$ combination of Real Fourier transform outperforms here. Kekre wavelet transform gives highest MAE value which indicates that it is not robust against cropping attack.

Figure9shows the performance of wavelet transforms in Gaussian noise attack (variance $=0.1$ ). Similar to contrast stretching attack, for higher scaling factor values, the distortion between watermarked image and noise added watermarked image is reduced. For scaling factor higher than 0.35 , all wavelet transforms except slant wavelet obtained from $(64,4)$ combination give very close MAE values. Slant wavelet generated from $128^{\star} 128$ and $2^{\star} 2$ size Slant matrices gives minimum distortion.

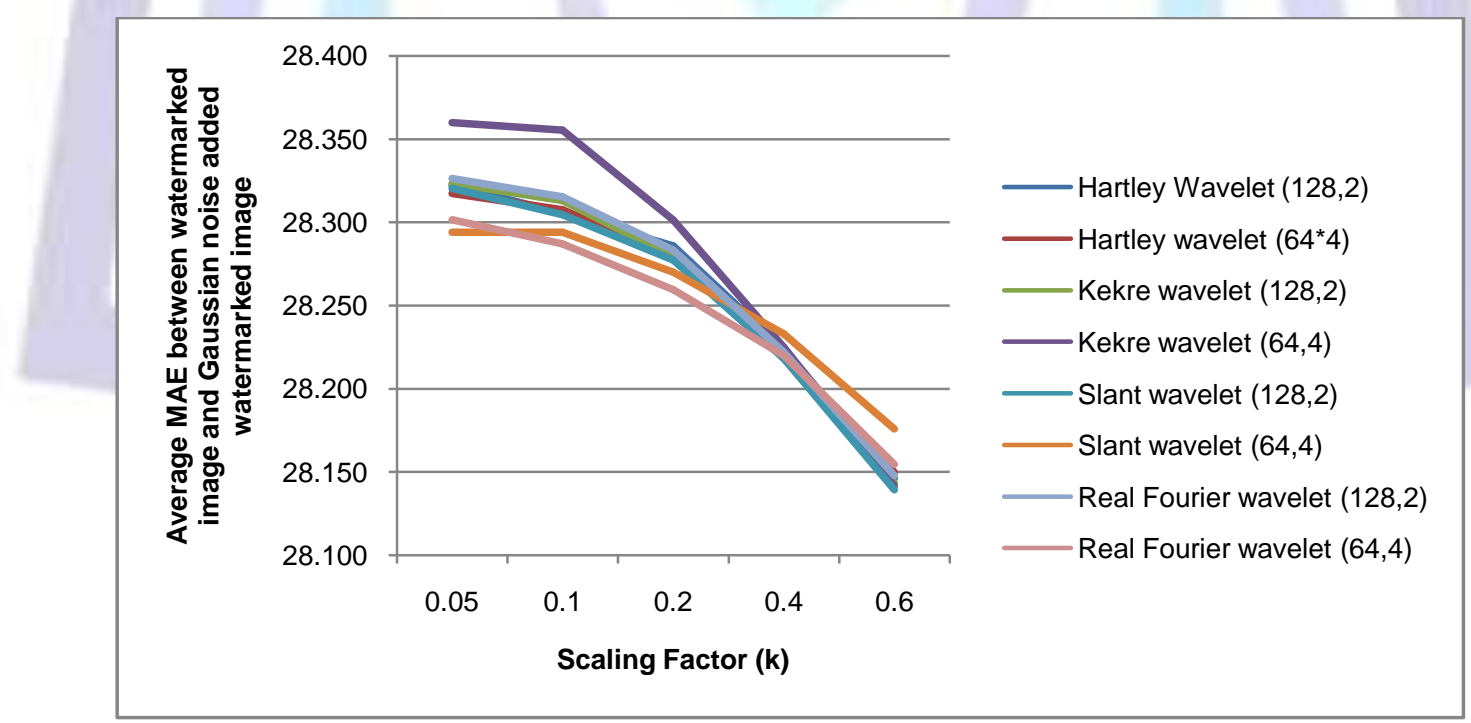

Figure 9. Average MAE between watermarked and Gaussian noise added watermarked image vs. scaling factor for different wavelet transforms 
Figure 10 gives the plot of scaling factor against Average MAE between original and extracted watermark for Gaussian noise attack.

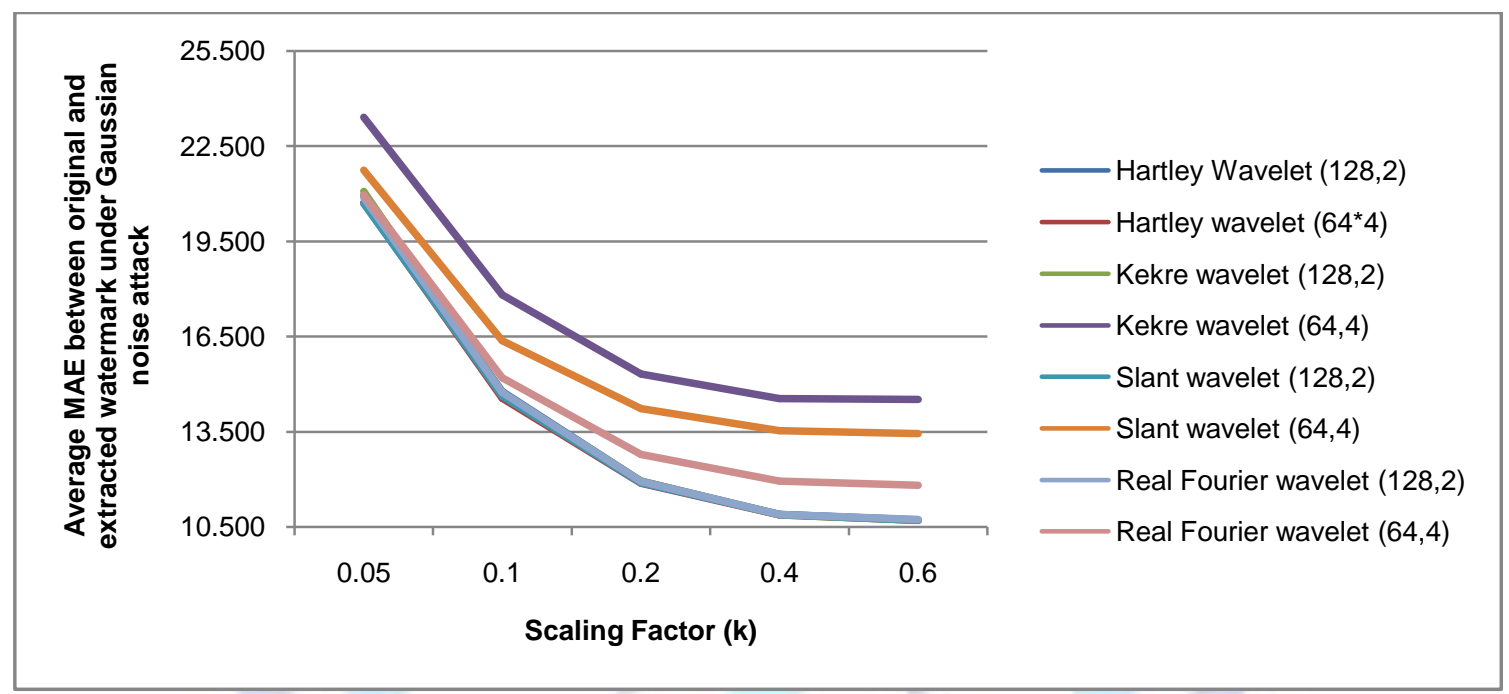

Figure 10. Average MAE between original and extracted watermark vs. scaling factor in Gaussian noise attack

From Figure 10, significant decrease in Average MAE values between original and extracted watermarks has been observed for increasing value of scaling factor k. Hartley wavelet obtained from $64^{*} 64$ and $4 * 4$ combination of Hartley transform and all wavelets of $(128,2)$ combination give closest values of MAE for all values of $k$.

Graph in Figure 11 shows the comparison of different wavelet transforms for histogram equalization attack. It is shown in terms of Average MAE between watermarked image and histogram equalized watermarked image against scaling factork. Almost every wavelet transform shows reduction in average MAE value with increase in scaling factor $k$. all wavelet transforms obtained from $(128,2)$ combination and Hartley wavelet, Kekre wavelet and Real Fourier wavelet obtained from $(64,4)$ combination give almost equal and better performance for $\mathrm{k}$ ranging from 0.2 to 0.6 .

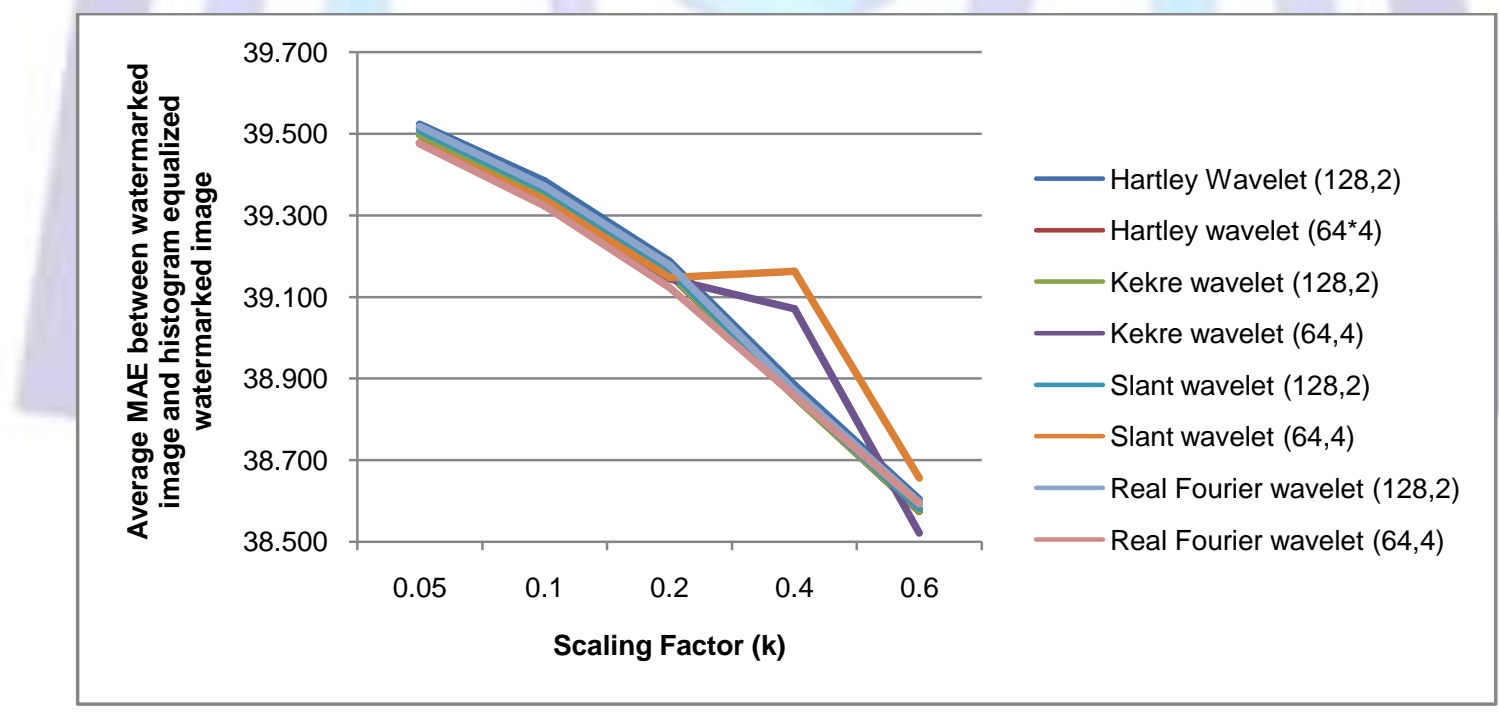

Figure 11. Average MAE between watermarked image and its histogram equalized version for various values of scaling factor

Figure 12 shows the graph of Average MAE between original and extracted watermark against scaling factor for histogram equalization attack with different wavelet transforms. For higher values of $\mathrm{k}$ ranging from 0.175 to 0.6 , Real Fourier wavelet obtained from $128^{*} 128$ and $2 * 2$ Real Fourier transform gives the smallest average MAE among all wavelet transforms. Performance of Real Fourier wavelet transform is also better than DCT wavelet and Walsh wavelet transforms presented in [19] and [20]. 


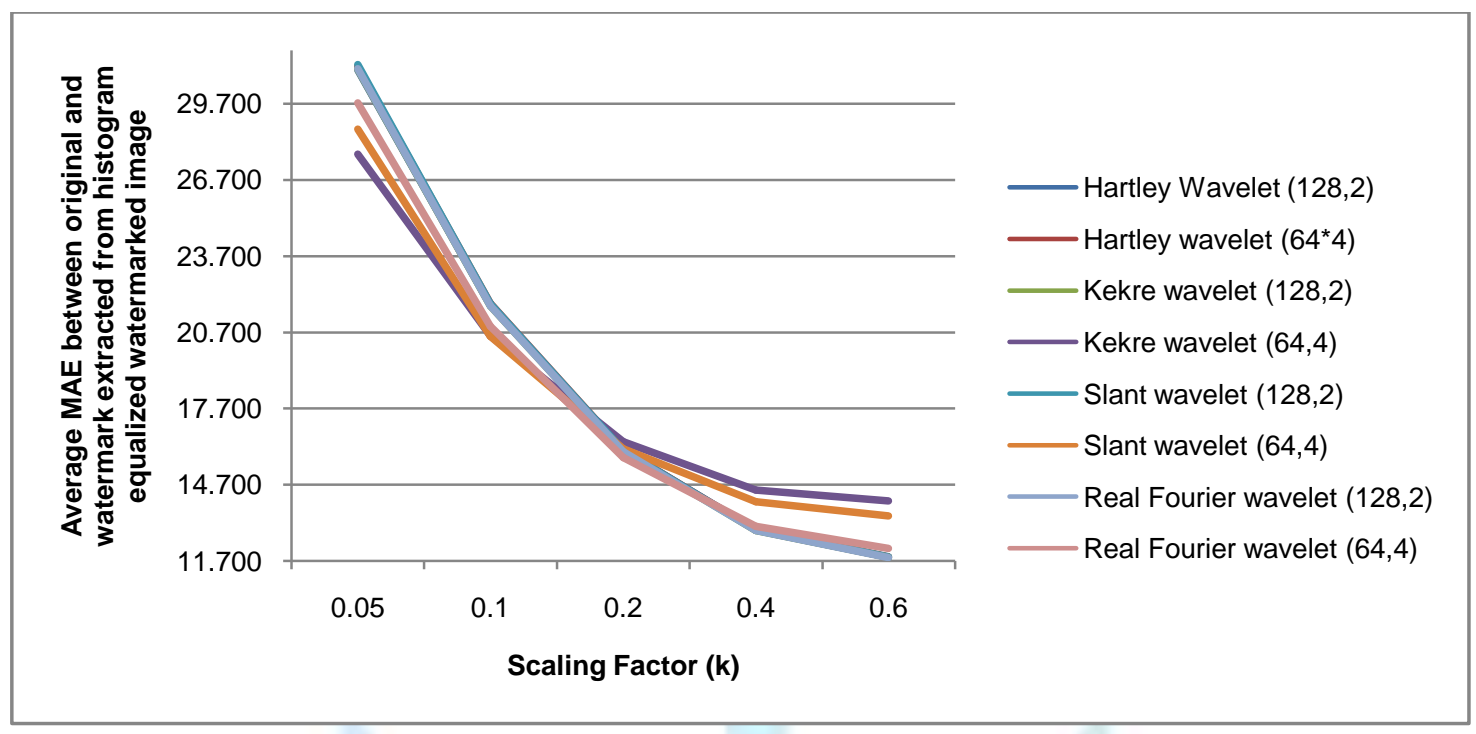

Figure 12. Average MAE between embedded and extracted watermark vs. scaling factor for histogram equalization attack

Figure 13 shows the average MAE between watermarked and resized watermarked image. Similar to image cropping attack, MAE increases with increase in scaling factor value. Thus with higher value of scaling factor used in embedding, more distortion is observed in host image. Slant wavelet generated from $64^{*} 64$ and $4{ }^{*} 4$ Slant matrices gives the smallest values of MAE as compared to any other wavelet transform for all values of k. Performance of this Slant wavelet is also better than DCT wavelet and Walsh wavelets presented in [19] and [20].

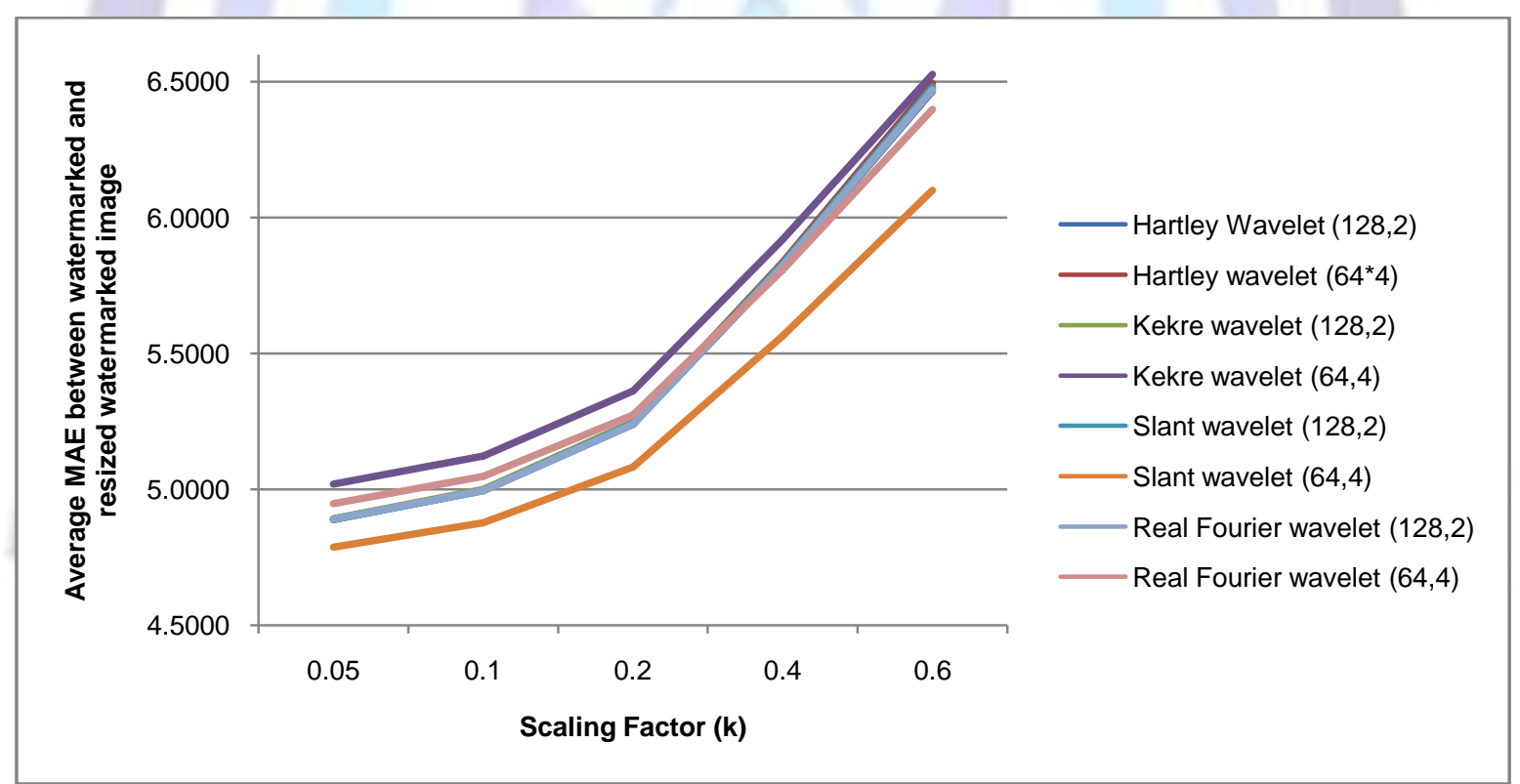

Figure 13. Average MAE between watermarked image and resized watermarked image vs. scaling factor for different wavelet transforms

Figure 14 shows comparison of different wavelet transforms for resizing attack. Average MAE between original watermark and watermarks extracted from resized watermarked image for different scaling factors are shown. For higher scaling factor values, extracted watermarks show high correlation with original watermark. For all wavelets, except Slant wavelet and Kekre wavelet of $(64,4)$ combination almost same performance has been observed. Amongst thembest results are observed for Kekre wavelet obtained from $(128,2)$ combination. These values are also lesser than DCT wavelet based watermarking and Walsh wavelet based watermarking presented in [19] and [20] respectively. 


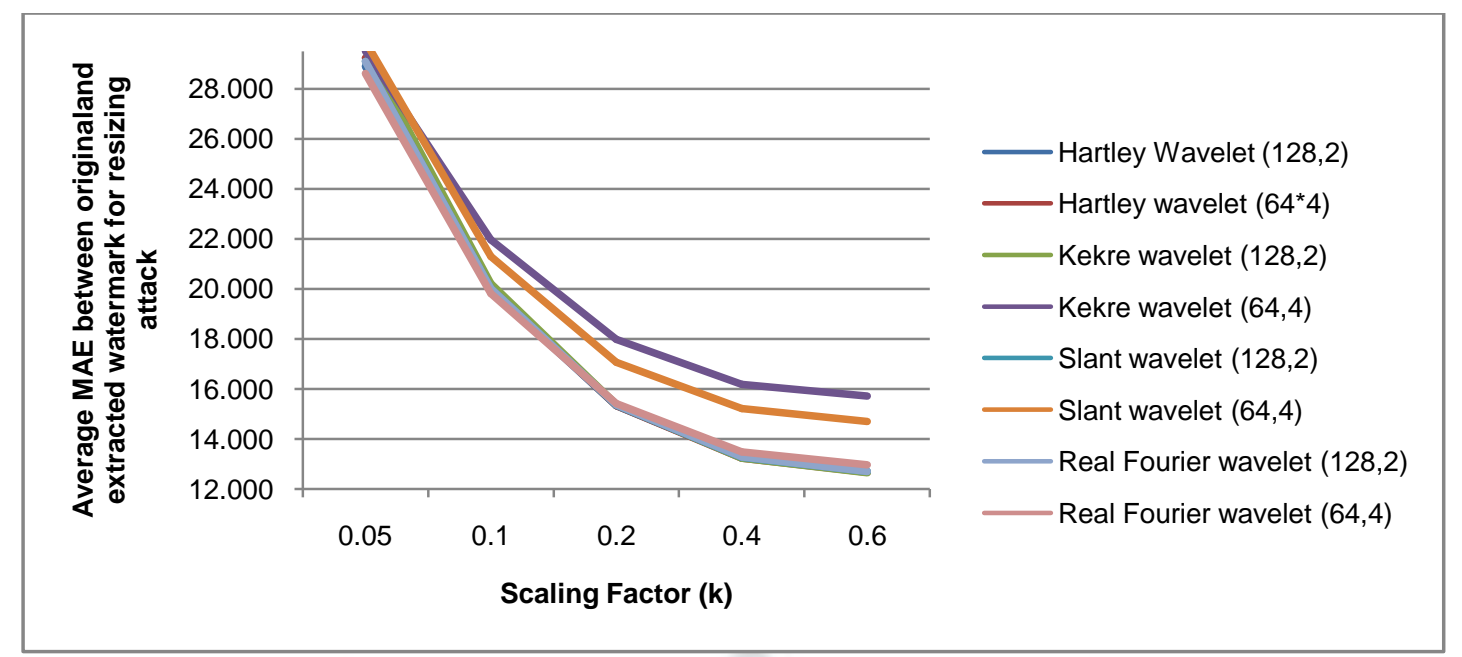

Figure 14. Average MAE between embedded and extracted watermark vs. scaling factor for resizing attack

\section{CONCLUSION}

Different wavelet transforms perform better for different attacks. Kekre wavelet transform gives better performance than other transforms namely Hartley wavelet, Slant wavelet and Real Fourier wavelet transforms for image resizing attack. Real Fourier wavelet transform outperforms in cropping and histogram equalization attack. Whereas performance of Hartley wavelet is better in contrast stretching and Gaussian noise attack. Except for cropping and resizing attacks, higher value of scaling factor leads to lesser distortion in attacked watermarked image. In general allwavelet transforms generated using $(128,2)$ size component orthogonal transforms give better performance than $(64,4)$ combination wavelets.

\section{REFERENCES}

[1]Gerardo Pineda Betancourth, Ayman Haggag, Mohamed Ghoneim, Takashi Yahagi, and Jianming Lu, "Robust Watermarking in The DCT Domain Using Dual detection", International Symposium on Industrial Electronics, IEEE 2006, pp. 579-584

[2] Hu Guan, Zhi Zeng, Shuwu Zhang, "A new DCT-based digital image watermarking algorithm", IEEE Proc. of International Conference on Automatic Control and Artificial Intelligence, 2012, pp. 166-169

[3] Jiang Yong Zheng, Dong Hong Liang, Jiang Zeng Liang, Miao Jin, "A DCT-BASED digital watermarking Algorithm FOR Image", IEEE Proc. of International Conference on Industrial Control and Electronics Engineering, 2012, pp. 12171220

[4]Cedillo-Hernandez, M.Garcia-Ugalde, F.J. ; Nakano-Miyatake, M. ; Perez-Meana, H.M., "DFT-Based Watermarking Method for Medical Images", IEEE Proc. of ninth International conference on Electronics, Robotics and Automotive Mechanics, 2012, pp. 21-24

[5] Chi-Man Pun, "A Novel DFT-based Digital Watermarking System for Images", IEEE Proc. of International Conference on Signal Processing, Volume 2, 2006

[6] Jianyong Huang, Changsheng Yang, "Image Digital Watermarking Algorithm Using Multiresolution Wavelet Transform", Proc. of IEEE International Conference on Systems, Man and Cybernetics, 2004, pp. 2977-2982.

[7] Nikita Kashyap, G. R. SINHA, "Image Watermarking Using 3-Level Discrete Wavelet Transform (DWT)", I.J.Modern Education and Computer Science, 2012, 3, pp. 50-56

[8] Dharm Singh, Naveen Choudhary, Madhuri Agrawal, "Scrambled Hybrid Wavelet-Singular Domain Digital Image Watermarking", Proc. of IEEE sixth International Conference on Software Engineering (CONSEG), 2012, pp. 1-6

[9] Mahendra M. Dixit, Paramhans K. Kulkarni, Pradeepkumar S. Somasagar, Veerendra C. Angadi, "Variable Scaling Factor based Invisible Image Watermarking using Hybrid DWT - SVD Compression - Decompression Technique", Proc. of IEEE Students' Conference on Electrical, Electronics and Computer Science, 2012, pp. 1-4

[10]Khaled Loukhaoukhaand Jean-Yves Chouinard, "A new image watermarking algorithm based on wavelet transform", IEEE Proc. of Canadian Conference on Electrical and Computer Engineering, 2009, pp. 229-234

[11]Yong-Gang Fu and Hui-RongWang, "A novel discrete wavelet transform based digital watermarking scheme", IEEE Proc. of $2^{\text {nd }}$ International Conference on Anti-counterfeiting, security and identification, 2008, pp. 55-58

[12]Qingtang Su, Xianxi Liu, WenchaoYang , "A Watermarking Algorithm for Color Image Based on YIQ Colour Space and Integer Wavelet Transform" 
[13]Ruth Buse Dili, Elijah Mwangi , "An image watermarking method based on the singular value decomposition and the wavelet transform", IEEE Proc. of International Conference on Image Analysis and Signal Processing, 2009, pp. 7073

[14]Gurparkash Singh Kang, "Blind Digital Image Watermarking Using Adaptive Casting Energy In Different Resolutions Of Wavelet Transform", IEEE Proc. of International Conference on Computer and Communication technology, 2010, pp.210-215

[15] H. B. Kekre, Tanuja Sarode, Prachi Natu, "Image compression using Real Fourier transform, its wavelet transform and hybrid wavelet with DCT", International Journal of Advances in Computer Science and Applications, Vol. 4 Issue 5,2013

[16] H.B.Kekre, Archana Athawale, Dipali Sadavarti, "Algorithm to Generate Wavelet Transform from an Orthogonal Transform”, International Journal of Image Processing, Vol.4, Issue 4, 2010, pp. 444-455.

[17]Dhananjay Theckedath, "Digital Image Processing using Matlab codes", Fourth edition, Nandu Printers and Publishers Private Limited, 2009

[18] H. B. Kekre, Archana Athawale, Dipali Sadavarti, "Algorithm to generate Kekre's wavelet transform from Kekre's transform", International Journal of Engineering Science and Technology, vol. 2(5), 2010, pp.756-767

[19]H. B. Kekre, Tanuja Sarode, Shachi Natu, "Hybrid watermarking of color images using DCT wavelet, DCT and SVD", International Journal of Advances in Engineering and Technology, Vol. 6, Issue 2, May 2013, pp. 769-779

[20] H. B. Kekre, Tanuja Sarode, Shachi Natu, "Robust watermarking using Walsh wavelet and SVD", International Journal of Advances in Science and Technology, Vol. 6 No. 4, April 2013

\section{Author's biography with Photo}

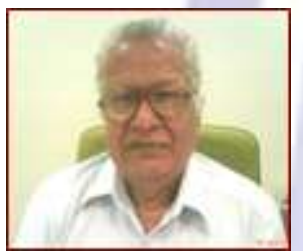

Dr. H. B. Kekre has received B.E. (Hons.) in Telecomm. Engg. from JabalpurUniversity in 1958, M.Tech (Industrial Electronics) from IIT Bombay in 1960,M.S.Engg. (Electrical Engg.) from University of Ottawa in 1965 and Ph.D.(System Identification) from IIT Bombay in 1970. He has worked Over 35 years as Faculty of Electrical Engineering andthen HOD Computer Science and Engg. at IIT Bombay. After serving IIT for 35 years, he retired in 1995. After retirement from IIT, for 13 years he was working as a professor and head in the departmentof computer engineering and Vice principal at Thadomal Shahani Engg. College, Mumbai. Now he is senior professor at MPSTME, SVKM's NMIMS University. He has guided 17 Ph.Ds, more than 100 M.E./M.Tech and several B.E. / B.Tech projects, while in IIT and TSEC. His areas of interest are Digital Signal processing, Image Processing and Computer Networking. He has more than 450 papers in National / International Journals and Conferences to his credit. He was Senior Member of IEEE. Presently He is Fellow of IETE, Life Member of ISTE and Senior Member of International Association of Computer Science and Information Technology (IACSIT). Recently fifteen students working under his guidance have received best paper awards. Currently eight research scholars working under his guidance have been awarded Ph. D. by NMIMS (Deemed to be University). At present seven research scholars are pursuing Ph.D. program under his guidance.

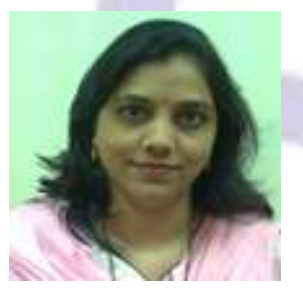

Dr. Tanuja K. Sarode has received M.E. (Computer Engineering) degree fromMumbai University in 2004, Ph.D. from Mukesh Patel School of Technology,Management and Engg. SVKM's NMIMS University, Vile-Parle (W), Mumbai, INDIA. She has more than 11 years of experience in teaching. Currently workingas Assistant Professor in Dept. of Computer Engineering at Thadomal Shahani Engineering College, Mumbai. She is member of International Association of Engineers (IAENG) and International Association of Computer Science andInformation Technology (IACSIT). Her areas of interest are Image Processing, Signal Processing and Computer Graphics.

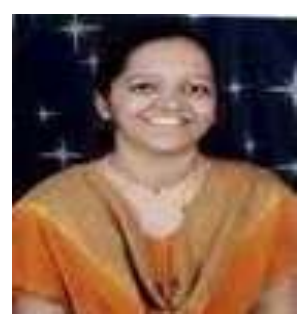

Ms. Shachi Natu has received M.E. (Computer Engineering) degree from Mumbai University in 2010. Currently pursuing Ph.D. from NMIMS University. She has 08 years of experience in teaching. Currently working as AssistantProfessor in Department of Information Technology at Thadomal Shahani Engineering College, Mumbai. Her areas of interest are Image Processing, Database Management Systems and Operating Systems. She has 12 papers inInternational Conferences/journal to her credit. 